\title{
Simulation study of a digital hydraulic independent metering valve system on an excavator
}

\author{
M. Sc. Miikka Ketonen, Adj. Prof. Matti Linjama \\ Laboratory of Automation and Hydraulics, Tampere University of Technology, Tampere, Finland \\ E-mail: miikka.ketonen@tut.fi, matti.linjama@tut.fi
}

\begin{abstract}
Independent metering valve (IMV) control of working hydraulic systems in mobile machines has been studied for more than two decades and during the past few years it has also been adopted to commercial excavators. The main advantages of the IMV systems, compared to load sensing and open centre systems are the possibility of optimizing the pressure losses of the metering edges and the possibility of re-routing the hydraulic energy between actuators. Energy re-routing can be realized without storing the energy to accumulators and thus avoiding the losses of additional routing valves and energy conversions. IMV combined with a hybrid system allows even more improved energy efficiency. Digital hydraulic IMV (D-IMV) allows additional benefits to the IMV systems with more fault-tolerant operation with robust components, faster and more precise control and leak-free valves.
\end{abstract}

The purpose of this study is to apply a D-IMV system to a midsize (20t) excavator and to study the differences in energy consumption of the working hydraulic system with four actuators. This paper presents the controller designed for the D-IMV and a simulation model for analysing the system. The energy consumption of the D-IMV is compared to the measured energy consumption of a state-of-the-art excavator with a load-sensing hydraulic system.

The Controller of the D-IMV system is realized with a sub-optimal mode control, so that instead of calculating and optimizing the total energy consumption of all possible mode combinations, simpler control logic is defined to remove the complex structure of the controller and ease the computational burden. Simulation study shows that the hydraulic input energy can be reduced $28-42 \%$ compared to a standard LS-controlled excavator. Detailed analysis of where the reduction emerges is presented.

Keywords: Digital hydraulics, Independent metering valve, mobile hydraulics, excavator

\section{Introduction}

Demands for the off-road machine builders by markets and legislations are driving new technologies to the traditionally conservative field of hydraulic engineering. Many of the construction equipment vendors have brought the hybrid systems to the markets and a few manufacturers are also utilizing independent metering valves (IMVs) in off-road machines. Excavators are the biggest machine type along with the wheeled loaders, which makes them perhaps the most important research subject in the field of mobile machines.

Most common state-of-the-art excavator valve systems are based on open centre flow control (OFC) or a load-sensing (LS) principle. There seems to be a trend that LS systems can be found in excavators below the 25 ton-class and above that the excavators have an open centre valve system. LS systems, and especially electronically controlled eLS systems, have proven to be more energy efficient, but have only become popular among European excavator manufacturers, while
Asian and American manufactures seem to favour open centre type valve systems. Main energy consuming features of current excavator valve systems are the throttling losses occurring when multiple actuators are used simultaneously and throttling losses occurring when an actuator operates far from the designed operation point due to the mechanically coupled inlet and outlet metering edges of the spool. Often it is considered a typical LS system lacks the operator feeling and response that operators tend to like.

Studies recently have also proposed many alternatives for the open centre and LS systems. These includes, for example flow demand-based eLS control systems [1], multiple constant pressure rail systems [2, 3], displacement control systems $[4,5]$ and different kinds of hydraulic hybrid systems $[6,7]$.

The advantage of an IMV-system compared to other technologies is the relatively simple system components. The system can be improved with only a single pump, keeping the manufacturing costs of the machine low. An IMV allows the 
hydraulic system to transfer hydraulic fluid between actuators and actuator chambers through the supply line or through the return line without additional routing valves. An IMV also can operate without additional pressure compensator valves. [8]

It is possible to implement the IMV technology to existing excavators with relatively small changes, and it can further improve energy consumption of LS systems by using differential and regenerative modes when the load force is suitable. An IMV also allows more flexibility in programming the boom control as requested by the user. $[8,9]$

For full performance, IMV systems require relatively high demands from the valves. Valves need to have high enough bandwidth, low pressure losses at high flows, bidirectionality, good repeatability and robust operation in changing environmental conditions. [10]

IMV systems are still used in only a few commercial applications; for example, the Caterpillar 336E H excavator, in which the IMV technology, or as they call it, the Adaptive Control System (ACS), is used together with a hydraulic hybrid system. [11]

\subsection{Digital hydraulic independent metering valves}

D-IMV systems consist of 4 independently controlled metering edges per actuator. Each of the metering edges consists of a series of parallel connected on/off-valves. The benefits of parallel connected digital valves, compared to traditional proportional valves, are the fault tolerance, the robustness and the fully repeatable and fast flow control. In some of the previous studies, a fifth flow metering unit between the A and B chambers also has been proposed, to give an extra degree of freedom for the system. [9]

In most cases, both IMV and D-IMV systems require pressure sensors in actuator ports, in the supply line and in the return line, and sophisticated control algorithms that estimate the load force and act upon it. Algorithms of IMV and D-IMV systems generally consist of the mode selection, and to calculate a target flow rate for each metering edge. [8]

\section{Digital hydraulic IMV in an excavator}

\subsection{Digital hydraulic valves for an excavator}

The D-IMV studied in this paper consists of four actuators: The boom, the arm, the bucket and the swing. Independent metering is realized with four flow metering units per actuator. The system layout is shown in Figure 1.

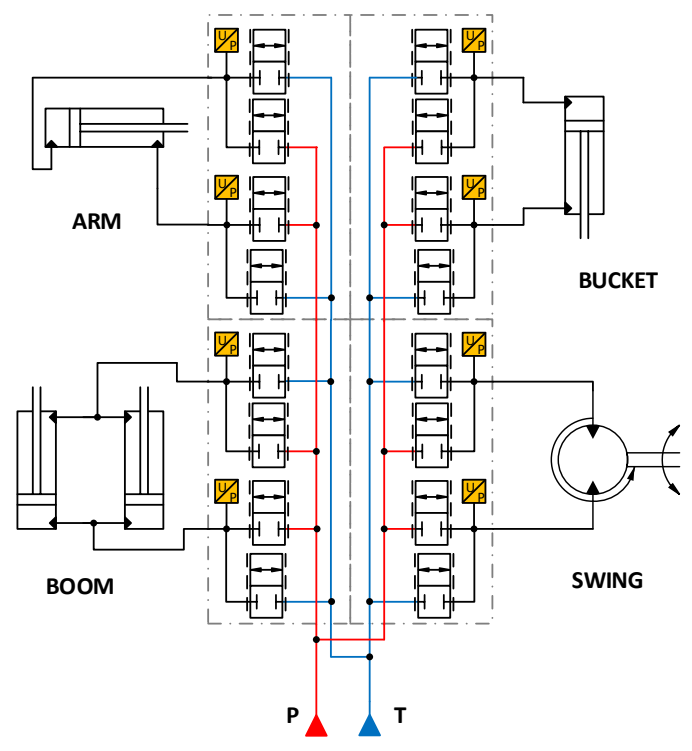

Figure 1. D-IMV valve system in excavator consisting of two pressure lines, 16 flow metering units and four actuators and chamber pressure sensors.

Pressure line P in Figure 1 is an electronically controlled LSpump pressure supply line and the pressure line $\mathrm{T}$ is a return line with a low pressure realized with a spring loaded check valve or a pressure relief valve. Each of the flow metering units, also known as digital flow control units (DFCUs), consists of seven on/off-valves with mixed PCM (Pulse Code Modulation) \& PNM (Pulse Number Modulation) coding of the valve sizes. In PCM coding, each valve has a different flowrate capacity, and the number of different available flowrate combinations is $2^{n}-1$, where $n$ is the number of valves. In PNM coding, each valve is equally sized. In practise, the flowrate capacity is adjusted by adding an orifice in line with part of the valves. Table 1 presents selected valve sizes for this study. [12]

Table 1. Selected flow rate series of each metering edge of the excavator. [l/min@0.5MPa]

\begin{tabular}{lccccccc} 
Valve: & 1 & 2 & 3 & 4 & 5 & 6 & 7 \\
\hline $\begin{array}{l}\text { Boom: PA-edge, PB-edge } \\
\begin{array}{l}\text { \& BT-edge. Arm \& Bucket: } \\
\text { all metering edges }\end{array}\end{array}$ & 35 & 35 & 35 & 17,1 & 8,7 & 4,3 & 2,2 \\
\hline $\begin{array}{l}\text { Boom: AT-edge, Swing: all } \\
\text { metering edges }\end{array}$ & 35 & 35 & 17,1 & 8,7 & 4,3 & 2,2 & 1,2
\end{tabular}

Figure 2 shows the control-flow characteristics of both of the control edge varieties presented in Table 1 . The number of different possible opening combinations varies from 68 to 96 . Control resolution, i.e., the relation between the maximum flowrate and the maximum flowrate step between two consecutive steps varies from 52.3 to 71.5. Resolution was selected based on simulation responses to handle cylinder motions without additional oscillation compared to the measured system and also based on available on/off valves. 


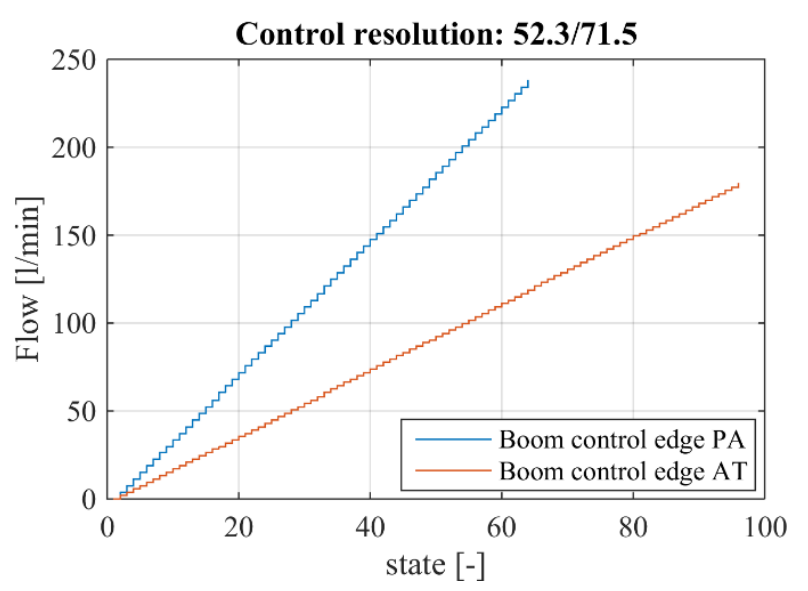

Figure 2. Possible flow rates of metering edges PA and AT of the boom at 1.5MPa.

\subsection{Operation modes of IMV}

The different modes are referred to as: PTe, PTr, TPe, TPr, PPe, PPr, TTe and TTr, in which the first letter describes whether the pump line $(\mathrm{P})$ or tank line $(\mathrm{T})$ is connected to the A-chamber, while the second letter describes which line is connected to the B-chamber. The third letter describes the direction of the movement, $\mathrm{e}$ for extending and $\mathrm{r}$ for retracting. Figure 3 presents the active metering edges with each mode.

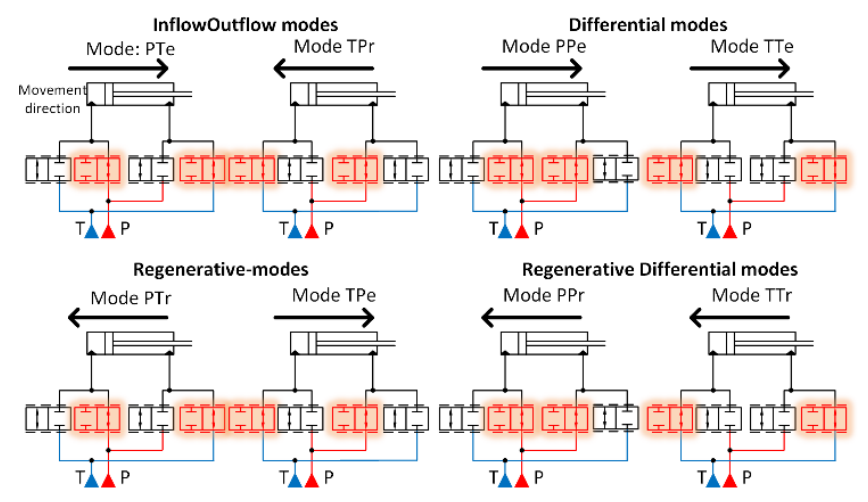

Figure 3. Different operation modes of the D-IMV system.

\section{Controller}

Many of the digital hydraulic valve controllers presented in earlier studies have been designed for powerful computers, such as the dSPACE Microautobox or PCs. More accurate and smoother control can be achieved if a more computationally demanding, model based valve control is used, but it has been shown in [13] that sufficient control for mobile application can be achieved also using simpler controllers that can be realized with commercially available controller units.

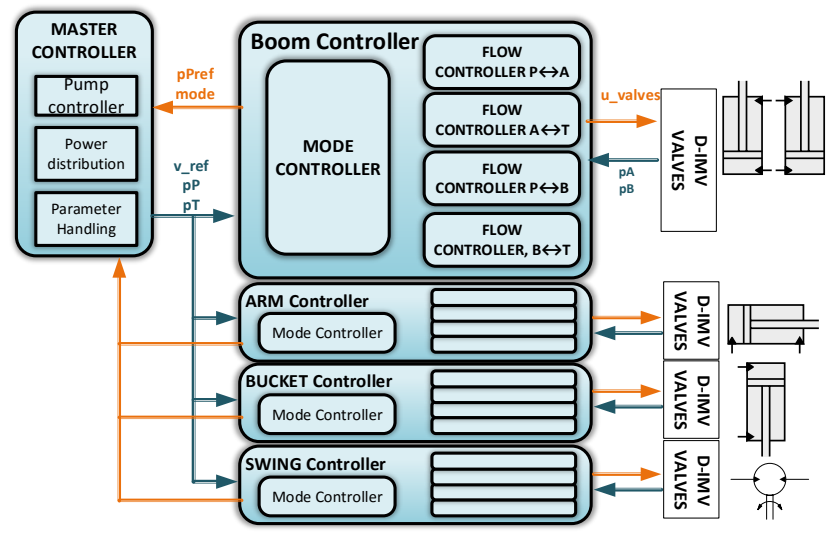

Figure 4. D-IMV controller structure.

The upper-level controller structure presented in Figure 4 includes four actuator controller subsystems and a master controller subsystem that includes the pump controller and the power distribution. Actuator controllers are identical to each other with a different set of parameters. Parameters are tuned in this study so that velocities and velocity oscillations are close to the measured responses of the reference excavator with LS-valve system.

\subsection{Master Controller}

The master controller structure is presented in Figure 5. Inputs to the controller are the measured supply line pressure $p P$ and the return line pressure $p T$, joystick signals and pump pressure requests from the actuator controllers. Outputs are the velocity references for the actuators, pump control signal and the filtered supply line and return line pressures for the actuator controllers. The Master controller realizes the eLS pump control by selecting the maximum of actuator supply pressure requests as a pump pressure reference and turns it into a control signal for the pump control valve. The Power and torque distribution block ensures that the engine will not stall and that the desired operation of actuators is ensured when the power demand exceeds the available hydraulic power.

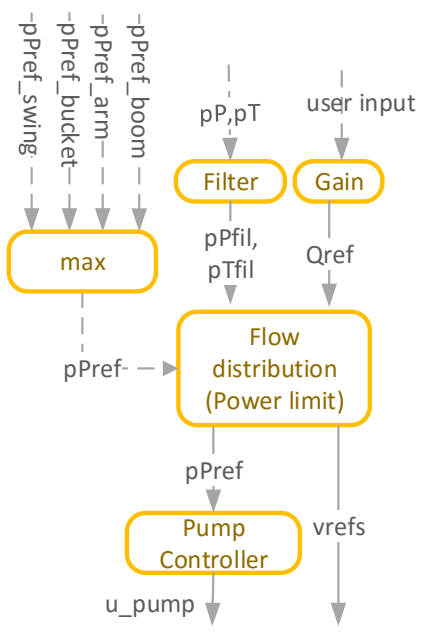

Figure 5. Master controller structure. 


\subsubsection{Power distribution and limitation}

In case the controller power demand or flow rate demand exceeds the machine limits, velocity references are lowered so that the total hydraulic power requirement is within the power limits of the system and maximum flow rate is not exceeded. In case the power demand exceeds the power limit, new velocity references for the actuators are calculated with an equation

$$
v_{\text {ref } f_{\text {new }}}=v_{\text {ref }} \frac{P_{\text {limit }}\left(n_{\text {engine }}\right)}{Q P_{\text {ref }} \cdot \max \left(p P_{\text {Refs }}\right)} W_{\text {dist }}
$$

where $v_{\text {ref }}$ is the user velocity reference, $P_{\text {limit }}\left(n_{\text {engine }}\right)$ is the available power with the current engine speed, $p P_{R e f s}$ is the vector of supply pressure references from the actuator controllers, $\mathrm{W}_{\text {dist }}$ is a weighting factor for the actuator to determine the priority of the actuator velocity reference and $Q P_{\text {ref }}$ is the total flow requirement. $\mathrm{W}_{\text {dist }}$ is calculated with equation

$$
W_{\text {dist }}=\frac{\overline{\boldsymbol{W}}_{\text {dists }, k}{ }^{v_{\text {ref }}} / \overline{\boldsymbol{v}}_{\text {maxs }, k}}{\sum\left(\overline{\boldsymbol{W}}_{\text {dists }} \overline{\boldsymbol{v}}_{\text {refs }} / \overline{\boldsymbol{v}}_{\text {maxs }}\right)} .
$$

where $\overline{\boldsymbol{v}}_{\text {refs }}$ is the vector of velocity references, $\overline{\boldsymbol{v}}_{\text {maxs }}$ is the vector of maximum actuator velocities and $\overline{\boldsymbol{W}}_{\text {dists }}$ is the vector including all four weighting gains of four actuators. By adjusting the relations between the gains, a desired relation of the actuator's velocities can be achieved. $Q P_{\text {ref }}$ is calculated as a sum of actuator flow rate demands, which is calculated using the equation

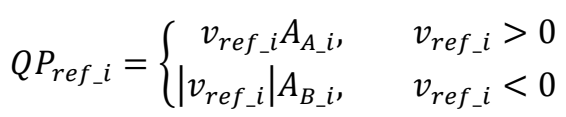

where $A_{A}$ and $A_{B}$ are the cylinder chamber areas or the radian chamber volumes of the swing motor.

If flowrate demand is exceeded, new velocity references are calculated with equation

$$
v_{\text {ref } f_{\text {new }}}=v_{\text {ref }} \frac{Q_{\text {limit }}\left(n_{\text {engine }}\right)}{Q P_{\text {ref }}} W_{\text {dist }}
$$

where $Q_{\text {limit }}\left(n_{\text {engine }}\right)$ is the available power with the current engine speed.

In the case of the swing actuator, desired operation is achieved also by limiting the swing torque. New pressure reference for the swing actuator is calculated with the equation

$$
\begin{aligned}
& p P_{\text {ref_sw_new }} \\
& =p P_{\text {ref_sw }} \frac{P_{\text {limit }}\left(n_{\text {engine }}\right)}{Q P_{\text {ref }} \cdot \max \left(p P_{\text {Refs }}\right)} W_{\text {dist }}
\end{aligned}
$$

To achieve equal operation in simulations and in the reference measurements, power distribution is limited by only limiting the swing torque. In case the boom, arm or bucket actuators have bigger pump pressure requests than the swing actuator, the swing torque cannot be limited and only the velocity reference of the swing actuator is reduced.

\subsubsection{Motion Controller}

In the simulation study, cylinder velocity references are derived from the position and velocity measurements of the reference machine. Reference velocities for the control system are calculated with a motion controller realizing the equation

$$
v_{\text {ref }}=K_{F F} v_{\text {meas }}+K_{P}\left(x_{\text {meas }}-x_{\text {sim }}\right)
$$

where $\mathrm{K}_{\mathrm{FF}}$ is feedforward gain and $\mathrm{K}_{\mathrm{P}}$ is feedback gain, $\mathrm{x}_{\text {meas }}$ is the reference cylinder position from the reference measurements and $\mathrm{x}_{\text {sim }}$ is the actuator position in simulations. The motion controller is only required for simulation purposes.

For the swing actuator, measured pilot pressures of the control valve in the reference measurement are used to define the start moment of the joystick actuation and a smooth trajectory going from 0 to 1 is applied to mimic the joystick control signal. Control duration is applied so that the steadystate swing positions are equal to the measured reference positions.

\subsection{Mode controller}

The mode controller structure is presented in Figure 6. The mode controller is built with a sub-optimal principle. It is known that typical pumps used in mobile machines have poor efficiency at high pressures and low flowrates, and that high pressures also tend to wear system components and thus to increase maintenance costs. The differential modes are not used to calculate the pump pressure reference, but are still used when possible to lower the pump flowrate demand.

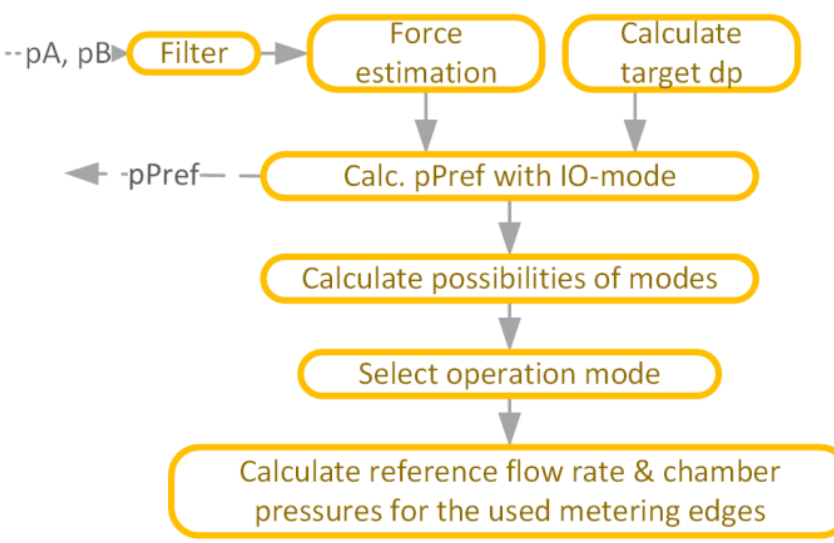

Figure 6. Mode controller flow chart.

Actuator force or torque is estimated from the measured chamber pressures and using a filter developed in [6]. This 
non-linear filter improves the dynamics of the force estimation, although a linear first-order filter works well in a simulation environment.

Most of the mode selection code is realized with a Matlab/Stateflow chart. The mode controller changes state from idle to running mode when $\left|v_{r e f}\right|$ is above the tolerance velocity $v_{t o l}$. If the actuator is moving, it goes to the idle mode when $\left|v_{\text {ref }}\right|<v_{\text {tol }} / 2$.

\subsubsection{Target pressure difference}

Target pressure difference over the valves is calculated as a function of the velocity reference $v_{\text {ref }}$ to allow minimum pressure difference during low and mid-high velocities, and increased cylinder velocity by increasing the pressure difference over the valves. There are two parameters for setting the target pressure difference: minimum pressure difference target $\left(d p_{\text {noml }}\right)$ and the maximum pressure difference target $\left(d p_{\text {nom } 2}\right)$. $d p_{\text {ref }}$ is calculated with equation

$$
\begin{aligned}
d p_{\text {ref }}=\max & \left(d p _ { \text { nom } 1 , \operatorname { m i n } } \left(d p_{\text {nom } 2},\right.\right. \\
& \left.\left.\left(\frac{\left|v_{\text {ref }}\right| A_{A}}{Q_{\text {dp1nom_max }}} \sqrt{d p_{\text {nom } 1}}\right)^{2}\right)\right)
\end{aligned}
$$

where $Q_{\text {dpInom_max }}$ is the flowrate when the valve metering edge $\mathrm{P} \leftrightarrow \mathrm{A}$ is fully open and the pressure difference is equal to $d p_{\text {noml }}$.

For the swing actuator sufficient acceleration is done by using dynamic target pressure difference calculation that increases the $d p_{\text {ref }}$ value when joysticks move rapidly. This will increase the pump supply pressure more rapidly when swing motion is started, and thus will improve the acceleration of the swing motion. For the swing motion, $d p_{\text {ref }}$ is calculated with the pseudo code:

$$
\begin{aligned}
& \text { if }\left(v_{\text {ref }}-v_{\text {ref_previous }}\right)>v_{\text {ref_diff_limit }} \\
& \quad \rightarrow d p_{\text {ref }}=\min \left(d p_{\text {nom } 2}, d p_{\text {ref_previous }}+\text { inc_rate }\right) \\
& \text { else if } d p_{\text {previous }}>d p_{\text {nom } 2} \\
& \quad \rightarrow d p_{\text {ref }}=\max \left(d p_{\text {nom } 1}, d p_{\text {ref_previous }}-i n c_{-} \text {rate } 3\right) \\
& \text { else } \\
& \quad \rightarrow d p_{\text {ref }}=d p_{\text {nom } 2}
\end{aligned}
$$

where $v_{\text {ref_diff_limit }}$ is the parameter describing how fast the joystick motion must be for starting to increase $d p_{\text {ref, }}$ and inc_rate is a parameter to adjust the length of the increased $d p_{\text {ref }}$ duration.

\subsubsection{Supply pressure reference}

The pump pressure reference $p P_{\text {ref }}$ is calculated for the standard inflow-outflow modes (IO-modes), first by calculating the required chamber pressures from the force/torque $(F)$ and given limitations. First, the outflow-side pressure is calculated with the equation:

$$
\begin{gathered}
p_{\text {out }}=\min \left(\operatorname { m a x } \left(p_{\text {min }}, p_{T}+d p_{\text {out }}\right.\right. \text { ratio } \\
\left.\left.\cdot d p_{\text {ref }}\right), p_{\text {max }}\right)
\end{gathered}
$$

where $p_{\min }$ and $p_{\max }$ are given lower and higher chamber pressure limits and $d p_{\text {out_ratio }}$ is a parameter to lower the outflow side target pressure difference to improve the energy efficiency.

Inflow-side chamber pressure is then calculated with the equation

$$
p_{\text {in }}= \begin{cases}\left(F+p_{\text {out }} A_{B}\right) / A_{A}, & v>0 \\ \left(F-p_{\text {out }} A_{A}\right) / A_{B}, & v<0\end{cases}
$$

where $A_{A}$ and $A_{B}$ are the piston areas or radian volumes of the motor. From these $p$ Pref is calculated with equation

$$
\begin{aligned}
p P_{\text {ref }}= & \min \left(\operatorname { m a x } \left(p P_{\min }, p_{\text {min }}\right.\right. \\
& \left.\left.+d p_{\text {ref }}\right), p P_{\text {max }}, p_{\text {in }}+d p_{\text {ref }}\right)
\end{aligned}
$$

where $p P_{\min }$ and $p P_{\max }$ are limits for the supply pressure.

\subsubsection{Calculation of possible modes}

After $p P_{\text {ref }}$ is calculated, the current possibility of each mode is checked. For the positive direction, the possibilities of modes are calculated with equations:

$$
\begin{aligned}
& P T_{-} \text {pos }=\left(p P_{\text {ref }}-p P\right)<0.5 d p_{\text {ref }} \\
& P T_{-} \text {notpos }=p P<\left(p P_{\text {ref }}-d p_{\text {ref }}\right) \\
& T P \_p o s=F<\text { force }\left(p T-d p_{r e f}, p P+0.5 d p_{\text {ref }}\right) \\
& T P \_ \text {notpos }=F>\text { force }\left(p T-d p_{r e f}, p P+d p_{r e f}\right) \\
& P P \_p o s=F<\text { force }\left(p P-2 d p_{r e f}, p P+d p_{r e f}\right) \\
& P P \_ \text {notpos }=F>\text { force }\left(p P-d p_{\text {ref }}, p P+d p_{\text {ref }}\right) \\
& T T \_p o s=F<\text { force }\left(p T-2 d p_{\text {ref, }} p T+d p_{\text {ref }}\right) \\
& T T_{-} \text {notpos }=F>\text { force }\left(p T-d p_{\text {ref }}, p T+d p_{\text {ref }}\right)
\end{aligned}
$$

where the force $(p 1, p 2)$ calculates the equation

$$
\operatorname{force}(p 1, p 2)=p 1 A_{A}-p 2 A_{B}
$$

For the negative directions the possibilities are calculated with equations:

$$
\begin{aligned}
& T P \_p o s=\left(p P_{\text {ref }}-p P\right)<0.5 d p_{\text {ref }} \\
& T P \_ \text {notpos }=p P<\left(p P_{\text {ref }}-d p_{\text {ref }}\right) \\
& P T_{-} \text {pos }=F>\text { force }\left(p P-2 d p_{\text {ref }}, p T-d p_{\text {ref }}\right) \\
& P T_{-} \text {notpos }=F<\text { force }\left(p P-d p_{\text {ref }}, p T-d p_{\text {ref }}\right) \\
& P P_{-} \text {pos }=F>\text { force }\left(p P+2 d p_{\text {ref, }} p P-d p_{\text {ref }}\right) \\
& P P \_ \text {notpos }=F<\text { force }\left(p P+d p_{\text {ref }}, p P-d p_{\text {ref }}\right) \\
& T T \_p o s=F>\text { force }\left(p T+d p_{\text {ref }}, p T-2 d p_{\text {ref }}\right) \\
& T T \_ \text {notpos }=F<\text { force }\left(p T+d p_{\text {ref }}, p T-d p_{\text {ref }}\right)
\end{aligned}
$$

\subsubsection{Mode selection}

The mode for extending motion is selected in the state machine presented in Figure 7. For the rectracting motion the princible is identical. For certain work cycles the controller cannot utilize all the modes, and thus the tables 
enable_ext_modes and enable_ret_modes are declared to allow only certain modes to be used. The logic of the state machine decides the best possible mode, or if none of the modes is possible, the logic selects the hold mode to wait for the proper supply line pressure.

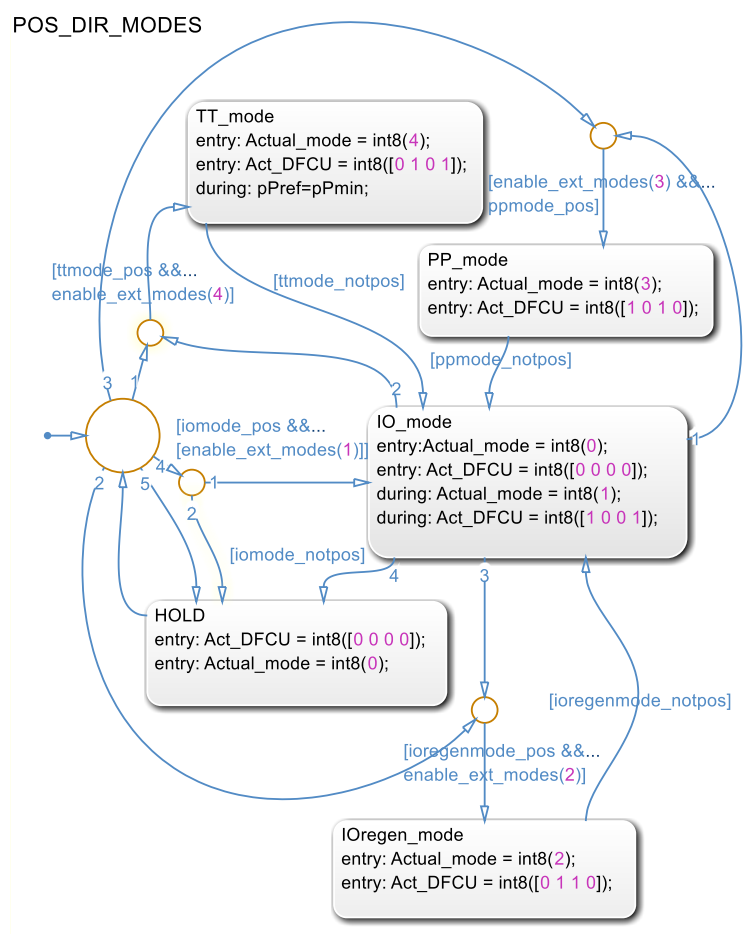

Figure 7. Mode selection in the state machine for positive direction.

\subsubsection{Calculation of reference values for the flow controller}

After the mode is selected, the controller selects which of the metering edges are to be used. For these metering edges, reference flowrates and chamber pressures are calculated for each valve.

Target flowrates are calculated for PA and AT-metering edges with equation

$$
Q_{\text {ref,PA/AT }}=v_{\text {ref }} * A_{A}
$$

and for metering edges PB and BT with equation

$$
Q_{r e f, P B / B T}=-v_{\text {ref }} * A_{B}
$$

Pressure references for the flow controller are set so that outflow-side is minimized, and maximum and minimum pressure limits are not exceeded. The inflow-side chamber pressure is set according to the current force level.

\subsection{Flow Controller}

The actuator controller includes a flow controller (Figure 8) for each metering edge, to calculate the optimal opening of the digital hydraulic valves. When the mode selection algorithm sends the flow requirement and target pressures for the active metering edges, the flow controller calculates the flowrates for each valve with the equation

$$
\begin{aligned}
& Q_{\text {valve }_{i}}\left(p_{1}, p_{2}\right) \\
& =\left\{\begin{array}{cc}
K_{1 i}\left(p_{1}-p_{2}\right)^{x_{1 i},}, & b_{1 i} p_{1}<p_{2} \leq p_{1} \\
K_{1 i}\left[\left(1-b_{1 i}\right) p_{1}\right]^{x_{1 i},} & p_{2} \leq b_{1 i} p_{1} \\
-K_{2 i}\left(p_{1}-p_{2}\right)^{x_{2 i}}, & b_{2 i} p_{21}<p_{1} \leq p_{2} \\
-K_{2 i}\left[\left(1-b_{2 i}\right) p_{1}\right]^{x_{2 i},} & p_{1} \leq b_{2 i} p_{2}
\end{array}\right.
\end{aligned}
$$

where $K_{1 \mathrm{i}}$ is the flow coefficient, $x_{1 \mathrm{i}}$ is the exponent and $b_{1 \mathrm{i}}$ is the critical pressure ratio of a single valve in the flow direction $1 \rightarrow 2$, and $K_{2 \mathrm{i}}, x_{2 \mathrm{i}}$, and $b_{2 \mathrm{i}}$ are the parameters for the opposite direction. For the simulation study, exponent values of 0.5 and critical pressure ratios of 0.25 were used. Flow rates of the individual valves are presented in Table 1 in Chapter 4.

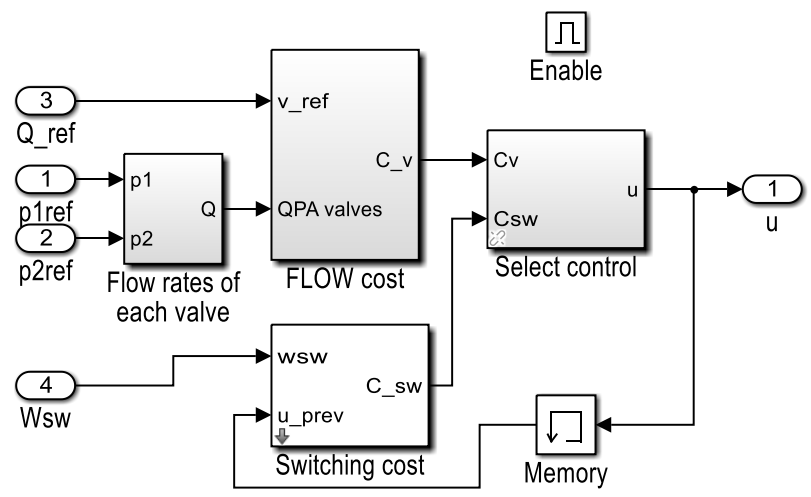

Figure 8. Flow Controller structure of Boom PA metering edge.

To avoid excessive valve switching, the final valve opening combinations are selected by minimizing the cost function, taking into account velocity error and the amount of valve state switching required. The valve opening combination is calculated with the equation

$$
\begin{aligned}
u_{\text {state }}=\min \left(\bar{v}_{\text {error }}\right. & \\
& +w_{\text {sw }}\left(\bar{Q}_{N O M}\right. \\
& \times \mid\left(\bar{u}_{\text {prev }} \times \bar{u}_{\text {ones }}\right. \\
& \left.\left.-\bar{u}_{\text {ctrl_mtrx }}\right) \mid\right)
\end{aligned}
$$

where $\bar{v}_{\text {errors }}$ is the array of the velocity errors for each valve opening combination, $w_{S w}$ is the weighting gain for the switching term, $\bar{Q}_{N O M}$ is a vector of nominal flow rates of the valves, $\bar{u}_{\text {prev }}$ is an array of the current state of valves, $\bar{u}_{\text {ones }}$ is an all-ones array with a length equal to the number of combinations and $\bar{u}_{\text {ctrl_mtrx }}$ is a matrix of valve states with each combination. 


\section{Simulation Model \& Reference Data}

The machine selected for comparison is a 21-ton LScontrolled excavator (Volvo EW210C, Figure 9). This excavator has a single pump for controlling all the actuators and the travel motors, and additional small auxiliary pumps for fan motor, pilot pressure supply and for other necessities. The excavator was instrumented with a pump flowrate sensor, actuator position sensors and actuator-chamber pressure sensors.

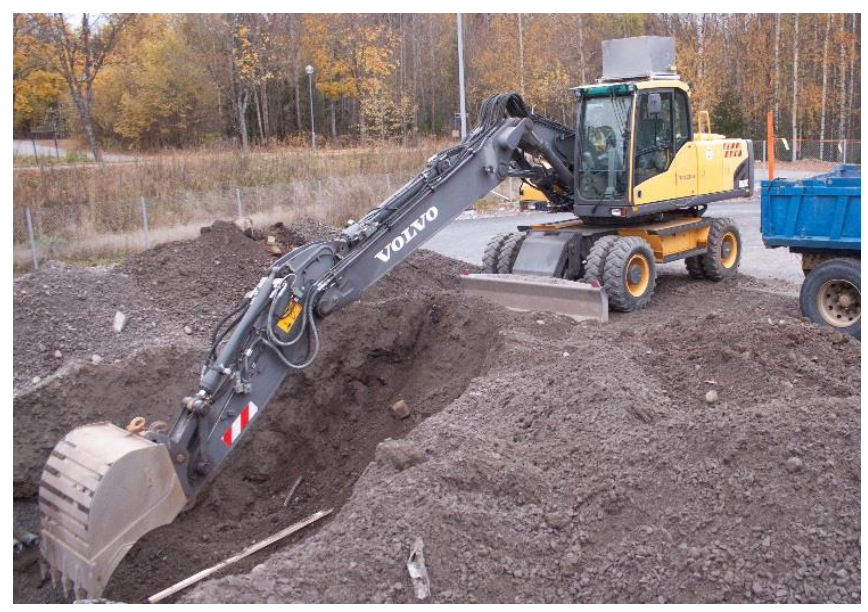

Figure 9. Volvo EW210C wheeled excavator used in reference measurements.

\subsection{Simulation model}

The simulation model (Figure 10) includes the D-IMV controller and the mechanical model of the excavator realized with Matlab Simulink. The mechanical model includes the inertias and kinematics modelled with SimMechanics, including the cross connection between the actuators. Supply pressure dynamics, control valves, hoses and actuators are modelled with Simulink. Model was verified to the measurement data from the excavator.

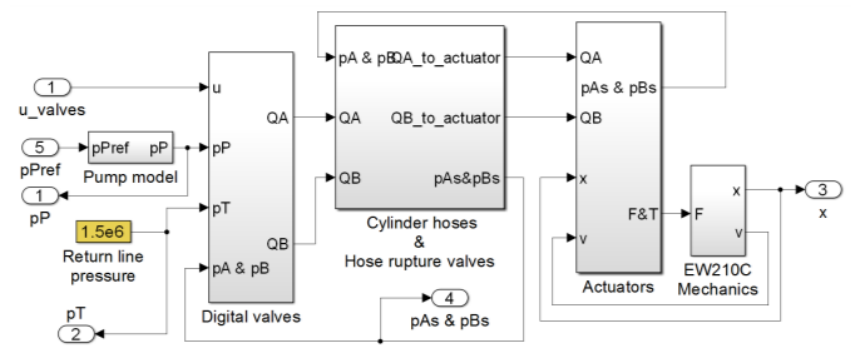

Figure 10. Hydraulic and mechanical model of the EW210C in Simulink.

The model of the digital valves includes four valve blocks, including $4 \times 7$ leak free on/off-valves per actuator. Flow rates of the on/off-valves are adjusted with orifices to accommodate the maximum velocity of the actuators and a control resolution that also allows low velocities.

The diesel engine is not modelled because the focus of the paper concentrates on the hydraulic efficiency comparison.
The pump is modelled to have typical dynamics for an open circuit mobile pump, but the efficiency of the pump is neglected because it is out of the scope of this study. The return line is modelled as a constant pressure line.

\subsection{Reference Data}

Reference data include measurements of a simulative 90degree truck loading cycle and earth grading cycle that are based on a JCMAS H 020 standard (Figure 11). The standard defines trajectories for comparing fuel consumptions of different sized machines in real life-like movements without touching the earth. This standard is selected to avoid the complex modelling of the earth and to realize as uniform conditions as possible for repeatable measurements, although it is does not match exactly the real world test cycles.

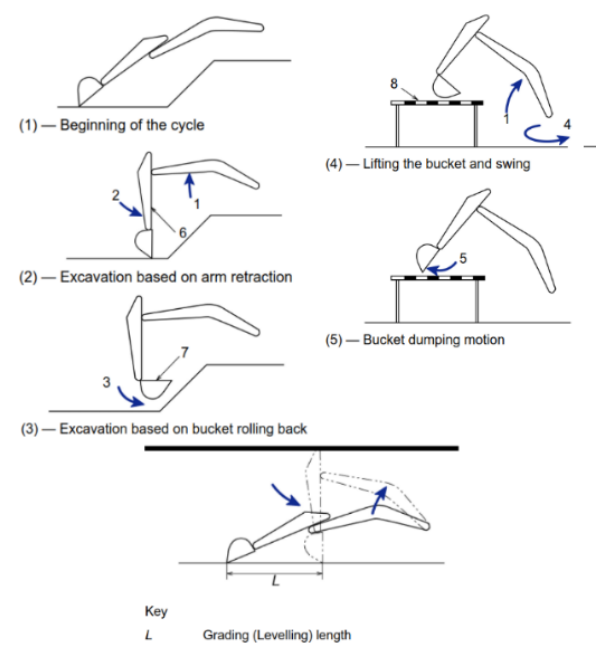

Figure 11. Truck loading cycle phases 1-5 and the grading cycle according to JCMAS H020. [15]

\section{Simulation results}

\subsection{Truck loading cycle}

Figure 12 presents the actuator positions and velocities in a single work cycle. There are some differences in velocity curves, but the final positions of simulations and measurements match. The simulation tends to oscillate a little more when hitting the end of the actuators because of the lack of a cylinder end damping model. 

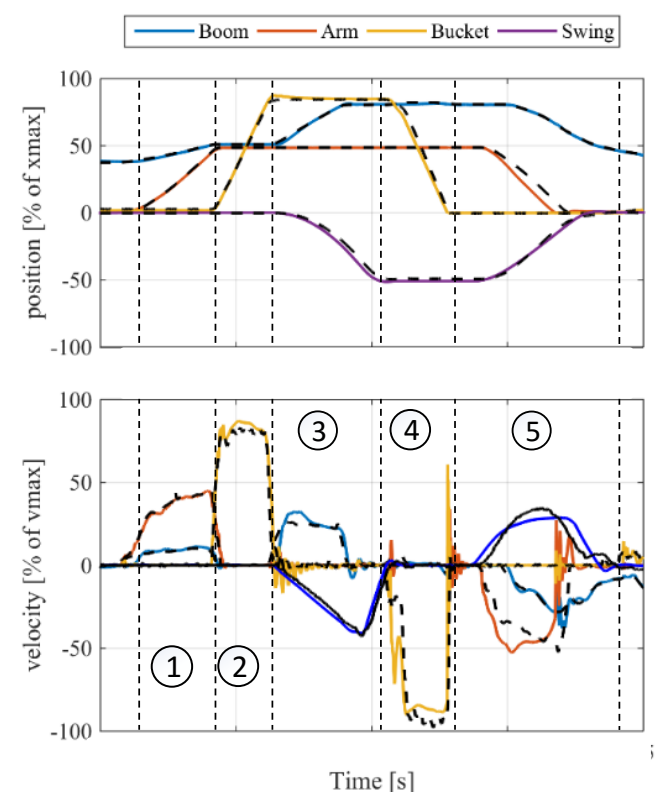

Figure 11. Positions and velocities from the reference measurements (dashed line) and from the simulated D-IMV system (solid lines) with different phases of the JCMAS digging cycle.

The upper graph of the Figure 13 presents the input and output powers of the simulated D-IMV system and the measured LS system, as well as the supply line pressure and flow rates. It can be seen that $19 \mathrm{~kJ}$ more work was done in the simulations than what was measured. This can be explained with a slight difference in velocity curves and in force/torque curves, but the relation to the input energy is so small that it can be considered negligible. Most of the difference comes from the behaviour difference of the swing drives. The peaks in the measured input power at phase 4 are due to the arm and bucket actuators hitting the end of the cylinder. The simulated system does not increase the cylinder force after hitting the end. In a truck loading cycle, a rapid force increase is not required when opening the bucket. The difference in the input energy comes from the lowered flow rate requirement because of the utilization of different modes.

Figure 14 presents the different modes used during the cycle. Phase 1 consists mostly of lifting the boom and extending the arm cylinder. As the boom lifting requires high pressure, the arm operates at differential modes and thus required input energy is reduced the around 40 percent. During the closing of the bucket in phase 2, the bucket cylinder is the only actuator and it operates in inflow-outflow mode. During the phase 3 , inflow-outflow modes are used by the boom and the swing actuator, and in phase 4 the bucket is opened in inflowoutflow mode. When returning to the starting position, the boom is lowered with the TTr-mode, in which zero pump flow is used simultaneously with the arm retraction and swing motion, which are done in inflow-outflow mode.
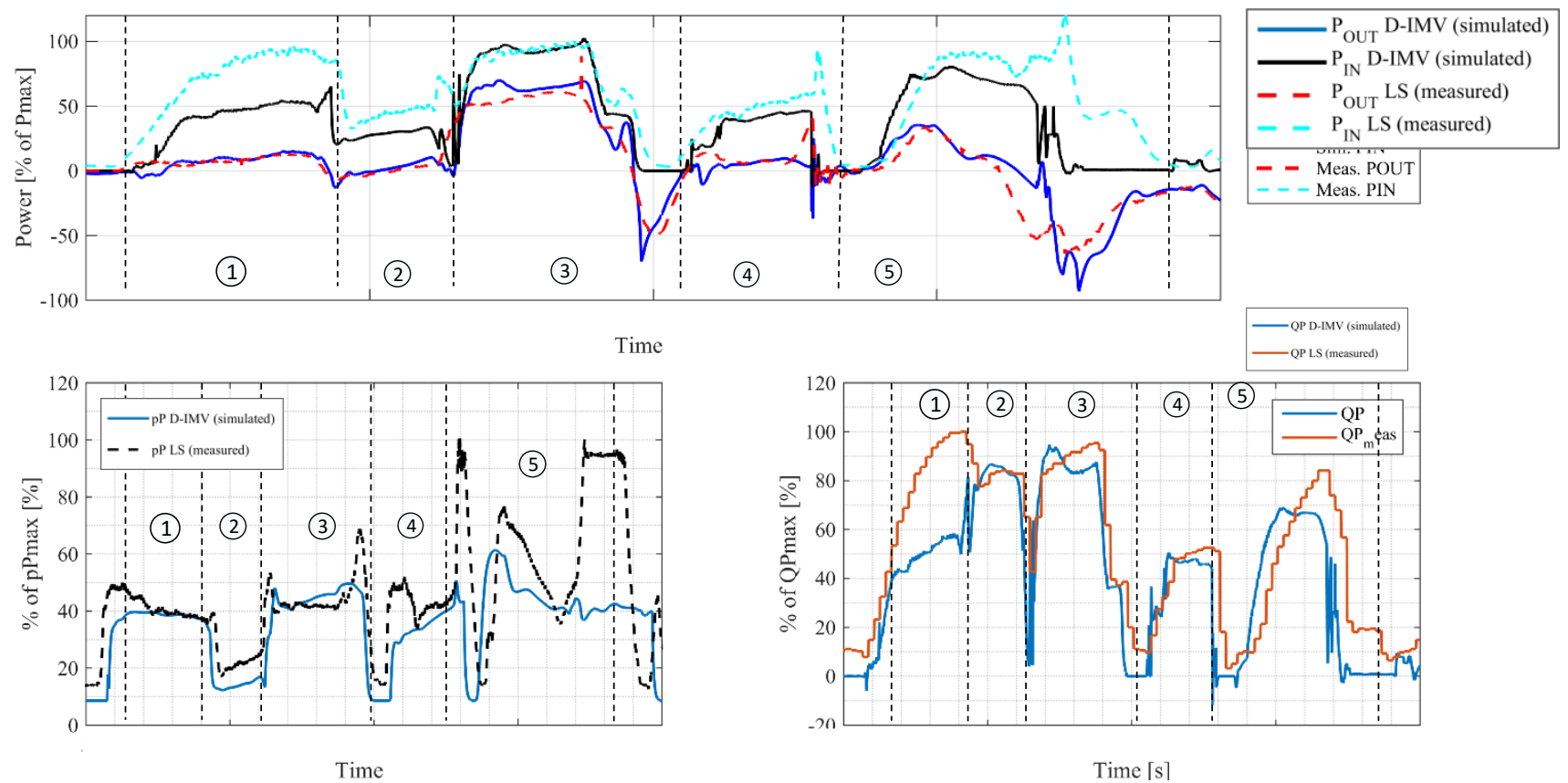

Figure 13. Hydraulic input powers $\left(P_{I N}\right)$ and mechanical output powers $\left(P_{\text {OUT }}\right)$, pump pressures and pump flow rates of the measured LS reference system (meas.) and the simulated D-IMV syste (sim.) in a JCMAS truck loading cycle (phases 1-5 divided in figure). 

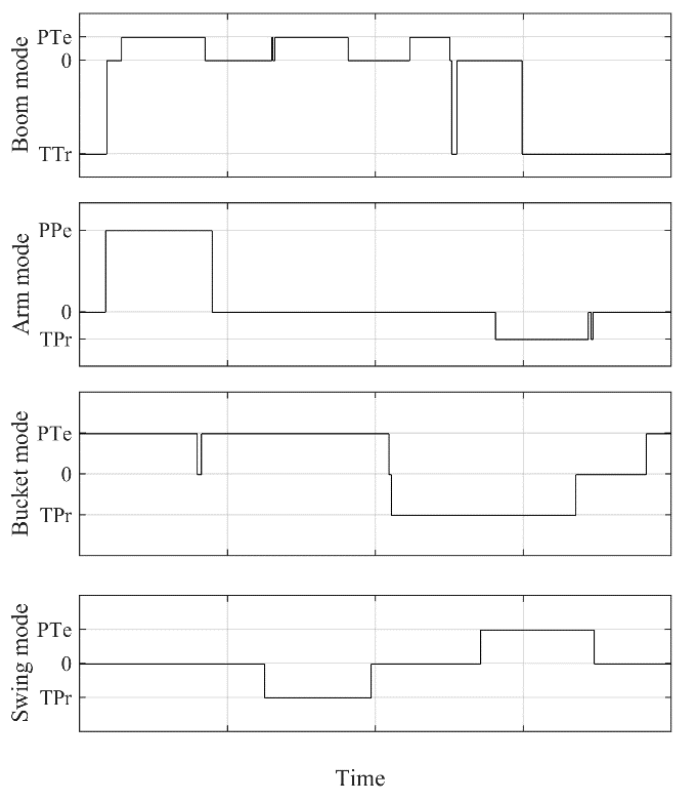

Figure 14. D-IMV Actuator modes in digging cycle simulations.

Figure 15 presents the cumulative input and output energies of four truck loading cycles. The total input energy was reduced 28 percent with D-IMV compared to the measured LS system.

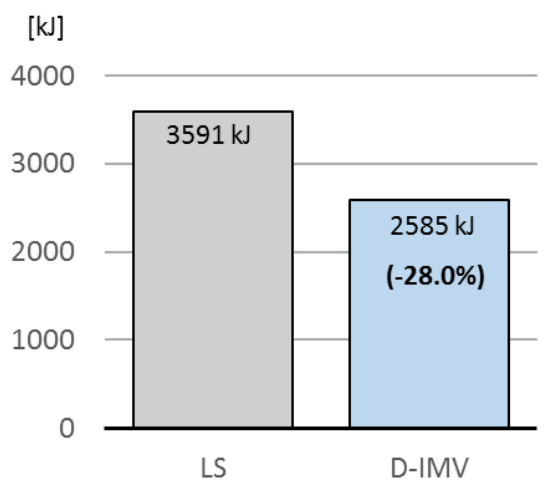

Figure 15. Measured and simulated cumulative energy consumptions in four consecutive truck loading cycles.

Total energy consumption of the working hydraulic circuit was reduced $28 \%$ in the digging cycle.

\subsection{Grading cycle}

Figure 16 presents the simulated and measured actuator positions, velocities and modes in a single grading work cycle. The bucket tip remains at a steady height from even ground surface, and the bucket is drawn from the out position to the in-position, and back.

Figure 17 presents the measured and simulated input and output powers, supply pressures and pump flowrates in the grading cycle. In the grading cycle, the main difference comes from the arm differential mode PPe used in the out-toin motion and the boom TTr-mode used in the in-to-out motion. Also, the metering losses are reduced during the into-out motion.
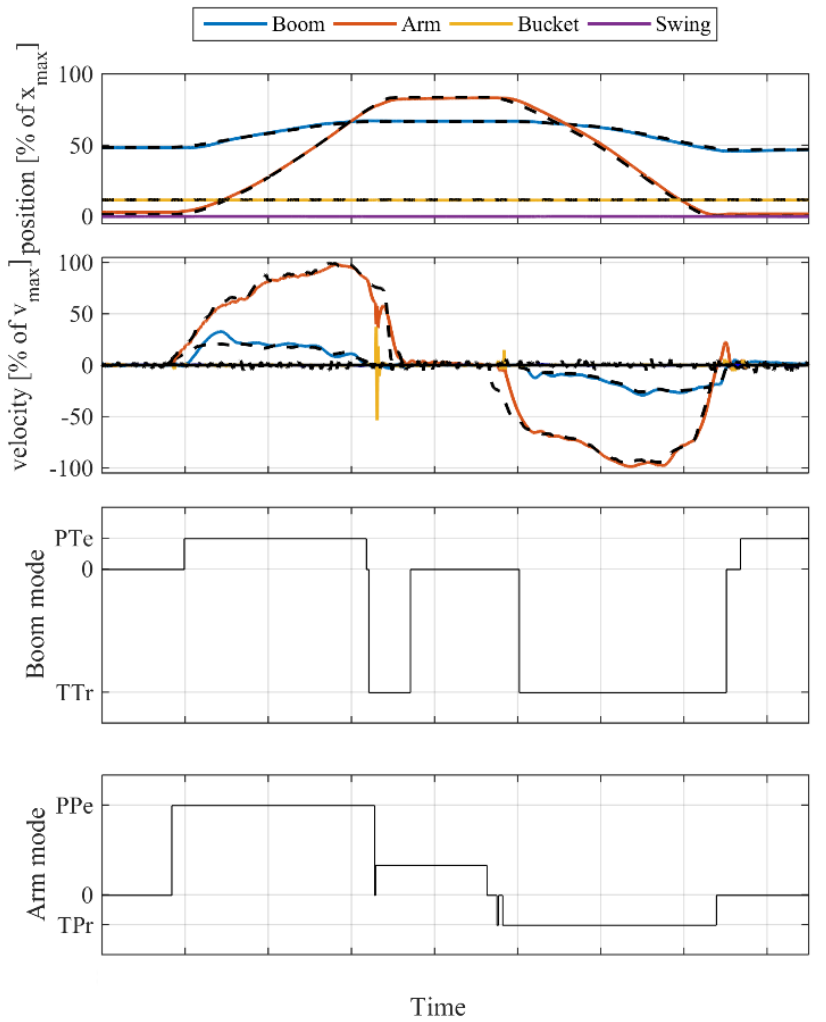

Figure 16. Simulated (D-IMV system) and measured (LS system) grading cycle position and velocities and modes used in simulation.

Figure 18 presents the cumulative input energies in four consecutive cycles. The total required energy was reduced $41.5 \%$ compared to the measured LS system.

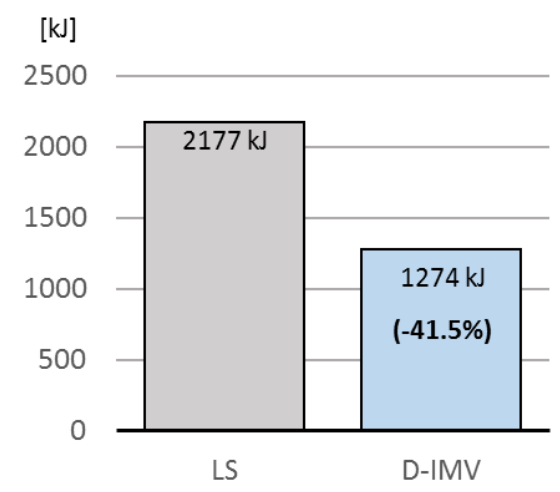

Figure 18. Measured and simulated cumulative input and output energies of four grading cycles. 

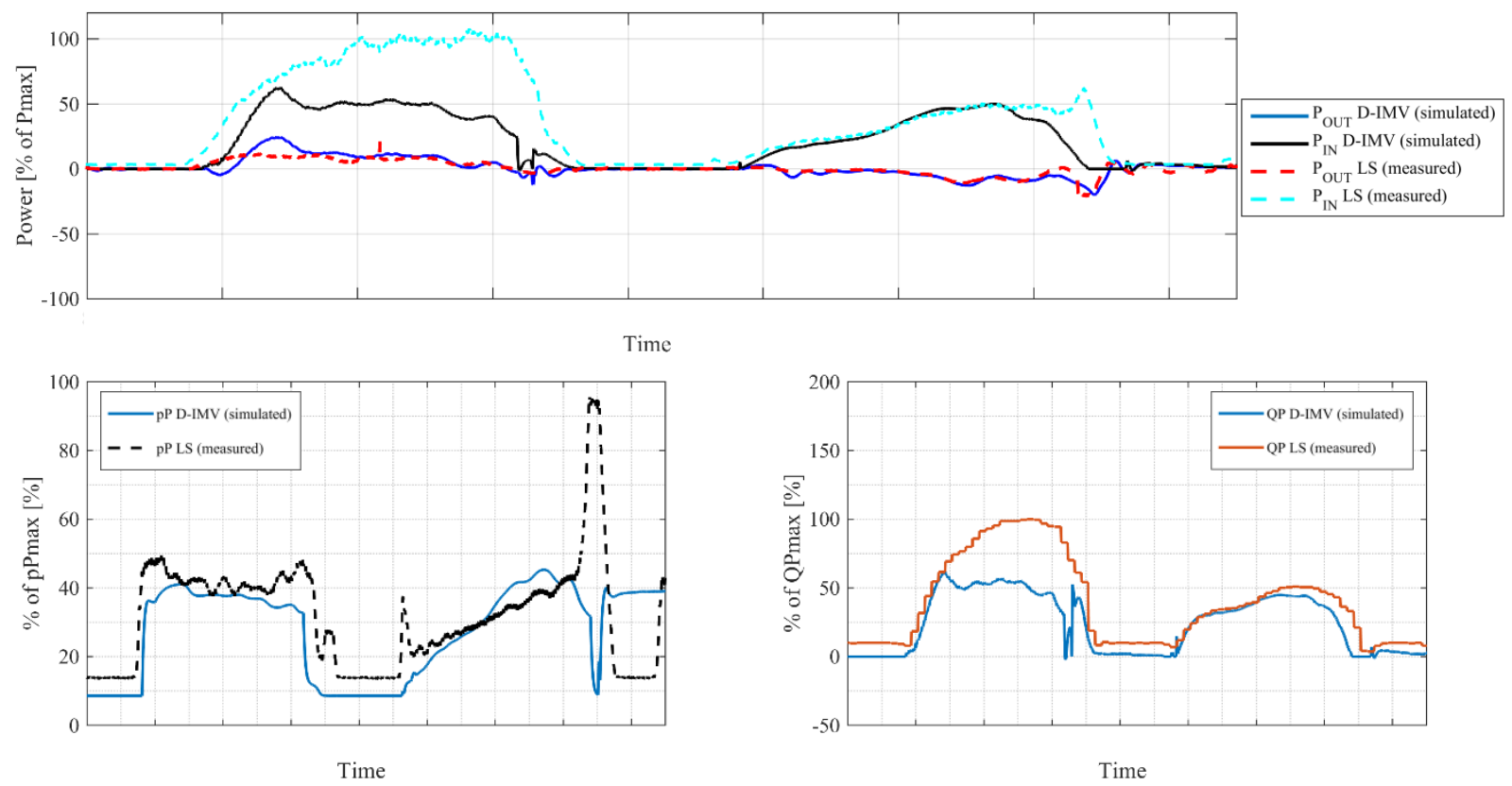

Figure 17. Hydraulic input powers (PIN) and mechanical output powers (POUT), pump pressures and pump flow rates of the measured LS reference system (meas.) and the simulated D-IMV system (sim.) in a JCMAS grading cycle.

\section{Conclusion}

D-IMV systems, and IMV systems in general, have great potential to improve the efficiency of mobile machines that often use working hydraulics with several actuators. This paper presented study comparing the measurement from a state-of-the art LS excavator and a simulated digital hydraulic IMV excavator. Comparison was done with a controller that utilizes different modes of the actuator, and thus lowers the flow rate required from the pump. The controller also keeps the supply pressure lower or at a similar level than that of current LS-systems.

In this study, system modifications other than those involving the valve system were kept to a minimum so as to compare the IMV system to a state-of-the-art LS-valve system in a 21ton excavator. The comparison included only hydraulic input energies, and it was shown that even without regenerative modes, hydraulic energy required for the two standard working cycles can potentially be decreased 28-42 percent.

Research continues in applying D-IMV technology to an actual test case to validate the results and study the system in actual work cycles.

\section{Acknowledgment}

This work was supported by the Doctoral School of Industry Innovations (DSII).

\section{References}

[1] Lettini, A., Havermann, M., Guidetti, M., \& Fornaciari, A. (2010). Electro-Hydraulic Load Sensing: a
Contribution to Increased Efficiency Through Fluid Power on Mobile Machines. Fuidotecnica, 345.

[2] Vukovic, M., Leifeld, R., and Murrenhoff, H., 2016. STEAM a hydraulic hybrid architecture for excavators. 10th International Fluid Power Conference (10. IFK), 810 March, Dresden, Germany.

[3] Linjama, M., Vihtanen, H-P., Vilenius, M. Secondary controlled multi-chamber hydraulic cylinder, The 11th Scandinavian International Conference on Fluid Power, SICFP'09, June 2-4, 2009, Linköping, Sweden

[4] K. Heybroek, G. Vael, and J.-O. Palmberg, "Towards resistance - free hydraulics in construction machinery," in The 8th International Fluid Power Conference, 8. IFK, Dresden, Germany, 2012.

[5] C. Williamson and M. Ivantysynova, "Power optimization for multi-actuator pump- controlled systems," in The 7th International Fluid Power Conference, 7. IFK, Aachen, Germany, 2010.

[6] K. Einola and M. Erkkilä, "Dimensioning and control of a hydraulic hybrid system of a cut-to-length forest harvester," in The 9th International Fluid Power Conference, 9. IFK, Aachen, Germany, 2014

[7] Lin, T., Wang, Q., Hu, B., \& Gong, W. (2010). Development of hybrid powered hydraulic construction machinery. Automation in Construction, 19(1), 11-19. https://doi.org/10.1016/j.autcon.2009.09.005 
[8] Eriksson, B., Palmberg, J.-O., Individual metering fluid power systems: Challenges and opportunities. Proceedings of the Institution of Mechanical Engineers, Part I: Journal of Systems and Control Engineering, 225:196-211, 2011.

[9] Nielsen, B. (2005). Controller Development for a Separate Meter-In Separate Meter-Out Fluid Power Valve for Mobile Applications, 237. Available http://vbn.aau.dk/files/74051350/brian_nielsen.pdf

[10]Heybroek, K., Larsson, J., Palmberg, J.-O., Mode Switching and energy recuperation in open-circuit pump control. In The Tenth Scandinavian International Conference on Fluid Power, Tampere, Finland, May 21$232007 . \quad$ Available http://liu.divaportal.org/smash/get/diva2:133010/FULLTEXT01.pdf

[11] Caterpillar website, http://www.cat.com/en_ID/news/machine-pressreleases/cat-sup-174-sup336ehhydraulichybridexcavatordeliversnocompromis.ht $\underline{\mathrm{ml}}$, visited 10.1.2017

[12]Linjama, M., Digital fluid power - state of the art. In The Twelfth Scandinavian International Conference on Fluid Power, Tampere, Finland, May 18-20 2011, available: https://pdfs.semanticscholar.org/a1a3/afd27352191866b 5614af9abea93ff5a9cf7.pdf

[13]Huova, M., Ahopelto, M., Ketonen, M., Ahola, V., Linjama, M., Huhtala, K., Characteristics of digital hydraulics with commercial controllers, The Seventh Workshop on Digital Fluid Power, February 26-27, 2015, Linz, Austria.

[14] Karvonen, M. (2016). Energy Efficient Digital Hydraulic Power Management of a Multi Actuator System. (Tampere University of Technology. Publication; Vol. 1384). Tampere University of Technology.

[15] JCMAS H020. Japan Construction Mechanization Association. Standard for of excavator fuel efficiency measurement procedure. 2010. 